\title{
Speed and Vibration Performance as well as Obstacle Avoidance Performance of Electric Wheel Chair Controlled by Human Eyes Only
}

\author{
Kohei Arai ${ }^{1}$ \\ Graduate School of Science and Engineering \\ Saga University \\ Saga City, Japan
}

\author{
Ronny Mardiyanto \\ Department of Electric and Electronics \\ Institute Technology of Surabaya \\ Surabaya, Indonesia
}

\begin{abstract}
Speed and vibration performance as well as obstacle avoidance performance of the previously proposed Electric Wheel Chair: EWC controlled by human eyes only is conducted. Experimental results show acceptable performances of speed vibration performance as well as obstacle avoidance performance for disabled persons. More importantly, disabled persons are satisfied with the proposed EWC because it works by their eyes only. Without hands and finger, they can control EWC freely.
\end{abstract}

Keywords-Human Computer Interaction; Gaze; Obstacle Avoidance; Electric Wheel Chair Control

\section{INTRODUCTION}

The Electric Wheel Chair: EWC controlled by human eyes only is proposed previously. It works well in principle. Some experiments show acceptable performances previously. This paper describes the performance of speed control and vibrations as well as obstacle avoidance performance.

The proposed system consists of forward and backward looking Web cameras mounted glass and pocket PC that allows Bluetooth communications. Thus users can be moved using the system. Pocket PC can be communicated with not only with Input and Output devices but also the other pocket PCs mounted on the other Electric Wheel Chairs: EWCs so that created and updated map information can be shares with many EWCs. The system provides obstacle finding with forward-looking camera so that EWCs can avoid obstacles. Location information of obstacles is uploaded to the other EWCs through Bluetooth communications. Thus all the EWCs can be controlled safely avoiding obstacles with the shared map information.

The following section describes the proposed system followed by experiments for the proposed system in terms of control performance of EWC, in particular, obstacle avoidance performance.

\section{The Proposed CoMputer INPUT System WITH HUMAN EYES-ONLY}

\section{A. Hardware configuration}

Hardware configuration is shown in Figure1. The proposed system consists of (1) two Web cameras mounted glass, (2) Pocket PC, (3) Ultrasonic Sensor. One of two Web cameras looks forward and the other one looks backward (acquires users' eye image). The web camera used 1.3 Mega pixel OrbiCam (Visible camera) and The Pocket PC used Sony VAIO UX180P with Intel Solo Processor U1400 1.20GHz and 512 MB RAM. First camera is used for acquired eye behavior and other camera is used for detect the obstacle. The Electric Wheel Chair used Yamaha JW-I type.

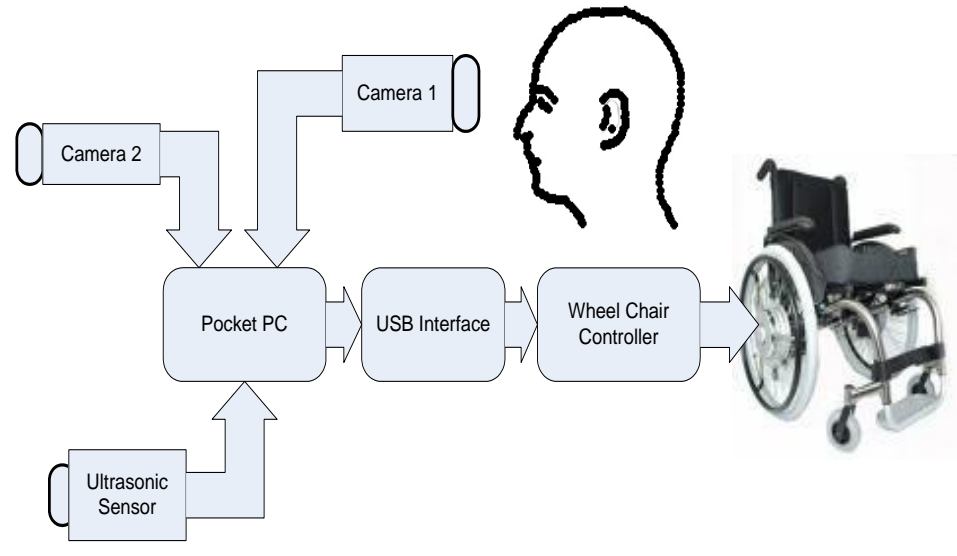

Fig. 1. Hardware configuration

\section{B. The method used and system parameters}

In order to control EWC, at least four keys, move forward, turn right, turn left and stop are required. For the safety reason, users have to look forward so that the key layout that is shown in Figure 2 is proposed.

\begin{tabular}{|c|c|c|}
\hline \multicolumn{3}{|c|}{ Stop } \\
\hline Turn left & Move forward & Turn right \\
\hline \multicolumn{3}{|c|}{ Stop } \\
\hline
\end{tabular}

Fig. 2. 3 by 3 of key layout for EWC control

Namely, key consists of 9 keys (3 by 3). Move forward and turn left/right are aligned on the middle row. Stop key is aligned on the other rows, top and bottom rows. Users understand the location of desired key so that it can be selected with users' eye-only. The backward looking Web camera whose resolution is 640 by 480 pixels acquires users' eye and its surrounding. Using OpenCV $\mathrm{C}^{(14)}$ of eye detection and tracking installed on the Pocket PC, users' eye is detected 
and tracked. If the OpenCV cannot detect users' eye, then EWC is stopped for safety reason. EWC is also stopped when users look at the different location other than the three keys aligned on the center row. When users are surprised human eyes used to be large. Such a situation can be detected with acquired image with backward looking Web camera so that EWC is stopped.

Intentional blink can be detected if the eye is closed for more than 0.4 seconds because accidental blink is finished within 0.3 seconds. In this connection, it is easy to distinguish between intentional and accidental blink. Also, key selection can be done every 0.4 seconds. Thus the system recognizes user specified key every 0.4 seconds. In order to make sure the user specified key, 10 frames per seconds of frame rate is selected for backward looking camera.

\section{Eye Detection and Tracking ${ }^{(17)}$}

Figure 3 shows the process flow of eye detection and tracking. Eye is detected by Viola-Jones classifier. The violaJones classifier employs ADABOOST at each node in the cascade to learn a high detection rate the cost of low rejection rate multi-tree classifier at each node of the cascade. To apply the viola-Jones classifier on the system, we use viola-Jones function in OpenCV ${ }^{(15)}$. Before use the function, we should create $\mathrm{xml}$ file data. The training sample (face or eye image) must be collected. There are two sample types: negative and positive sample. Negative sample correspond to non-object images. Positive sample correspond to object image. After acquired image, OpenCv will search the face center location and continue with search the eye center location. Advantage this function is fast and robust.
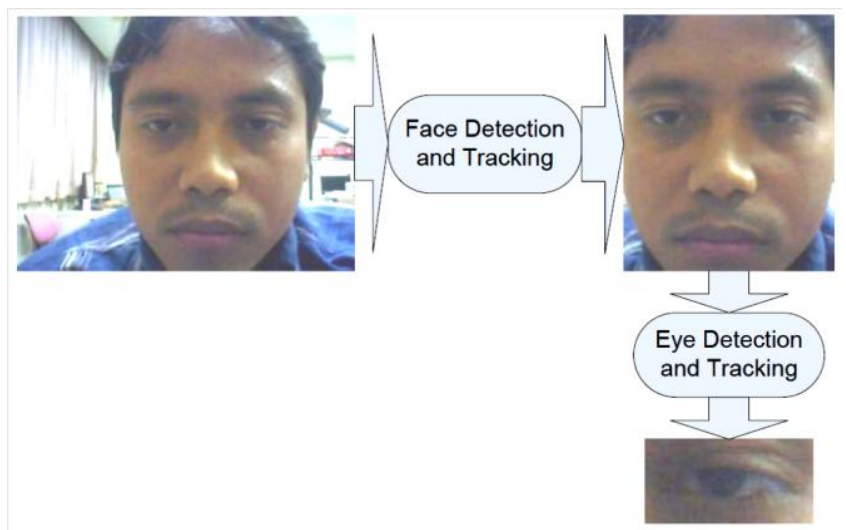

Fig. 3. Eye detection and tracking

\section{Template Matching}

Eye behavior is detected by template matching. Template matching which used is not based on histograms; the function matches an actual image patch against an input by sliding the patch over the input image. There are several template matching methods:

\section{1) Square difference matching methods}

These methods match the square difference, so perfect match will be 0 and bad matches will be large.

$$
\begin{aligned}
& R_{s q_{-} d i f f}(x, y)= \\
& \sum_{x^{\prime}, y^{\prime}}\left[T\left(x^{\prime}, y^{\prime}\right)-I\left(x+x^{\prime}, y+y^{\prime}\right)\right]^{2}
\end{aligned}
$$

This method will obtain good result only if both images have same pixel intensity. Because of output result only 0 and 1 , it not sophisticated for the proposed system.

\section{2) Correlation matching methods}

These methods multiplicatively match the template against the image, so a perfect match will be large and bad matches will be small or 0 .

$$
\begin{aligned}
& R_{c c o r r}(x, y)= \\
& \sum_{x^{\prime}, y^{\prime}}\left[T\left(x^{\prime}, y^{\prime}\right), I\left(x+x^{\prime}, y+y^{\prime}\right)\right]^{2}
\end{aligned}
$$

\section{3) Correlation coefficient matching methods.}

These methods match a template relative to its mean against the image relative to its mean, so a perfect match will be 1 and a perfect mismatch will be -1 ; a value of 0 simply means that there is no correlation.

$$
\begin{aligned}
& R_{c c o e f f}(x, y)= \\
& \sum_{x^{\prime}, y^{\prime}}\left[T^{\prime}\left(x^{\prime}, y^{\prime}\right) \cdot I^{\prime}\left(x+x^{\prime}, y+y^{\prime}\right)\right]^{2} \\
& T^{\prime}\left(x^{\prime}, y^{\prime}\right)= \\
& T\left(x^{\prime}, y^{\prime}\right)-\frac{1}{(w \cdot h) \sum_{x^{\prime \prime}, y^{\prime \prime}} T\left(x^{\prime \prime}, y^{\prime \prime}\right)} \\
& I^{\prime}\left(x+x^{\prime}, y+y^{\prime}\right)= \\
& I\left(x+x^{\prime}, y+y^{\prime}\right)-\frac{1}{(w \cdot h) \sum_{x^{\prime \prime}, y^{\prime \prime}} I\left(x+x^{\prime \prime}, y+y^{\prime \prime}\right)}
\end{aligned}
$$

In this system we used Correlation coefficient methods and give additional normalized to reduce the effects of lighting difference between the template and the image.

$$
\begin{aligned}
& z(x, y)= \\
& \sqrt{\sum_{x^{\prime}, y^{\prime}} T\left(x^{\prime}, y^{\prime}\right)^{2} \cdot \sum_{x^{\prime}, y^{\prime}} I\left(x+x^{\prime}, y+y^{\prime}\right)^{2}}
\end{aligned}
$$

Result values for this method that give the normalized computation are:

$$
R_{\text {ccoeff }_{\text {norm }}}(x, y)=\frac{R_{\text {ccoeff }}(x, y)}{z(x, y)}
$$

In this system, four template images are used to determine eye gaze. These images are acquired during calibration step. The best result will obtain eye gaze. The threshold is set to 90\% match. 


\section{E. Calibration Step}

Eye gaze measurements that apply template matching methods will does work on fix illumination and fix condition. Illumination changes, shadow, different of eye shape when it is used by other users, and others difference condition will obtain mismatch result.

This problem can be solved by calibration step. The calibration step consists of acquiring template image and self evaluation as is shown in Figure 4.

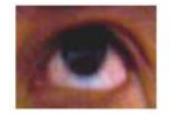

Up

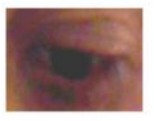

Down

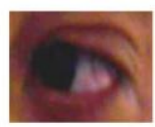

Left

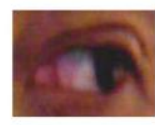

Right
Fig. 4. Result of calibration step. These steps obtain up, down, left, and right image. Up image is used for move forward, down is used for stop, left is used for turn left, and right is used for turn right

System will acquire eye image when looking at down, up, left, and right keys. Next, system will evaluate template image. If template image is good template, it will be used. If not, system will acquire eye image again until good template are obtained. When system is started, it will check that template images are proportional. Illumination, shadow, and eye shape will compare with current eye image. If there are not proportional image, calibration step will be ran.

\section{F. Custom Microcontroller}

Yamaha JW-I Electric wheel chair type is used. To control EWC using Pocket PC, custom microcontroller circuit is used. USB interface on pocket PC is used to connect with other peripheral. The custom microcontroller circuit is use RS232 communication. Microcontroller AT89S51 type is used. To connect between pocket $\mathrm{PC}$ and custom microcontroller circuit, USB to Serial communication is used. The microcontroller will drive relay to move the EWC. Microcontroller connection is shown in Figure 5.

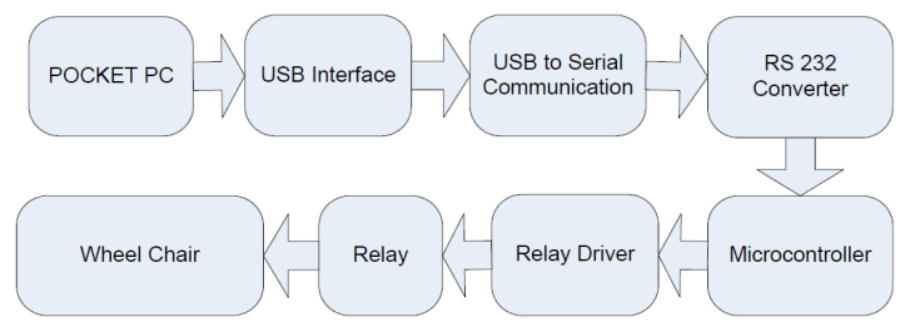

Fig. 5. Microcontroller AT89S51 connects to other peripheral through serial communication. Serial communication type should be converted to USB communication using USB to serial converter.

\section{G. Obstacle Avoidance}

In order to safety reason, obstacle avoidance system is implemented in our system. Obstacle avoidance system will able to identify the obstacle in front of EWC and avoid it. This system consists of two approaches: (1) Obstacle detection, and (2) Best Path Finding.

\section{H. Obstacle Detection}

Obstacle detection is consisting of image processing based and ultrasonic sensor based. Image processing based utilizes background subtraction between current image $\mathrm{I}(\mathrm{x}, \mathrm{y})$ and background image $\mathrm{B}(\mathrm{x}, \mathrm{y})$. Background subtraction method will obtain black-white image $\mathrm{S}(\mathrm{x}, \mathrm{y})$ which represent obstacle. On this image, obstacles appear as white pixel. By using searching of outer line from white pixels, we can determine position and size of obstacle.

$$
\begin{aligned}
& S(x, y)=0, \text { if }|I(x, y)-B(x, y)|<\text { threshold } \\
& S(x, y)=255, \text { if }|I(x, y)-B(x, y)|<=\text { threshold }
\end{aligned}
$$

Weakness of background subtraction method is working only if two images have same position of translation, scale, shear, and rotation. To solve these problems, we utilize affine transformation. This transformation requires 3 points that appears on both images as is shown in Figure 6.

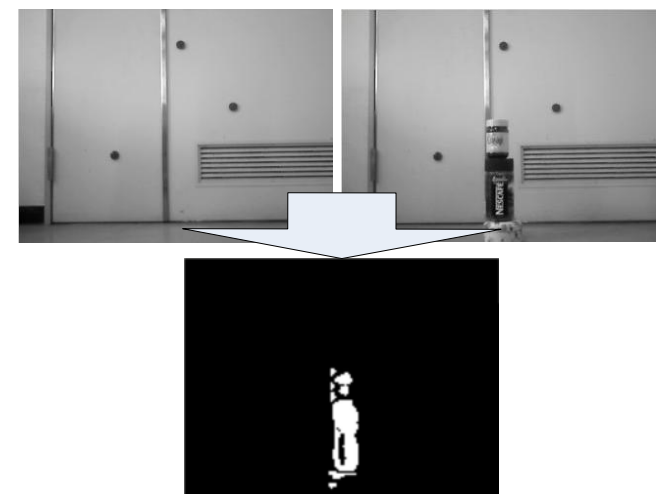

Fig. 6. Background subtraction method, left-top is background image and right-top is current image. Background subtraction method will obtain blackwhite image (bottom)

Translation, scale, share, and rotation parameter can be determined from these points. Affine transformation required at least three noticeable important points. These points should be appears on both images. It can be detected by several ways: corner, edge, specific object, and etc. Corner and edge have many points and its will create computation problem. In our system, we decide to use specific object. The specific object can be an easily recognized object, text character, chessboard wall, and etc. Flow process of obstacle detection using affine transformation is shown in Figure 7.

The specific object will obtain one coordinate from center of area. Obstacle detection using affine transformation requires identifying at least three kind of object, so it will obtain three noticeable important points.

First step of obstacle detection is convert source image into gray image. By using template matching, system will find specific object position. Using six noticeable points, system calculate translation, share, scale, and rotation parameter. These parameters will used for creating affine matrix. 


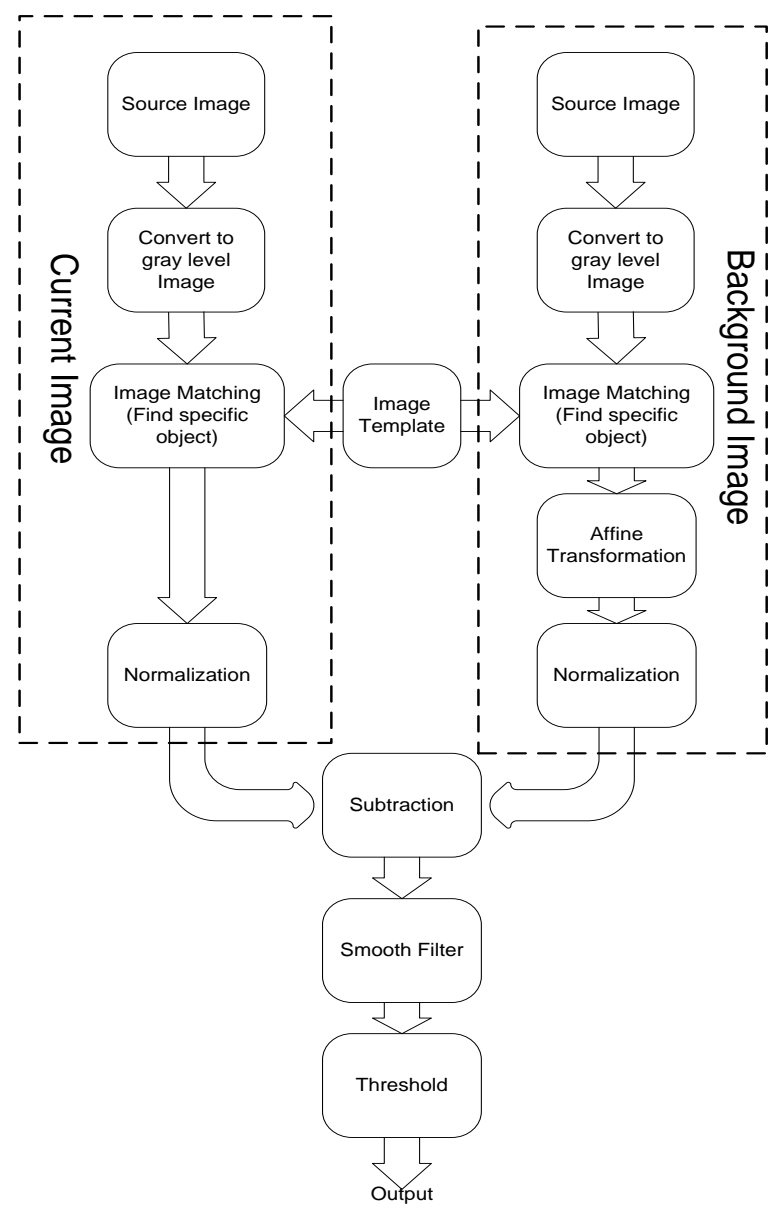

Fig. 7. Obstacle Detection. This methods rely on subtraction between two images and affine transformation.

Affine matrix apply on background image will obtain affine image. Normalization is used to eliminate disturbance such shadow, noise, and etc. Next, subtract between output form current image and background image. Smooth filter is used to reduce noise which is caused by subtraction process. Last step is applying threshold on image and it will obtain black and white image. Obstacle will be signed as group of white pixel. After black white image is obtained, we should return center of white pixel area into current coordinate and coordinate of obstacle is founded. Because of so many type of specific object, we got best performance of specific object by using chessboard wall. The advantage of the chessboard (Figure 8 ) is easily detected and robust on distance changes. We use 3 types of chessboard: 3 by 4,3 by 5 , and 3 by 6 as are shown in Figure 9. The other obstacle detection is use ultrasonic sensor. This sensor has advantage when visual system does not work. In case EWC move into surrounding glass door, smoke condition, and minimum lighting will caused visual system of obstacle detection fail. Ultrasonic sensor consists of transmitter and receiver part. We use ultrasonic sensor with $40 \mathrm{kHz}$ frequency resonance.
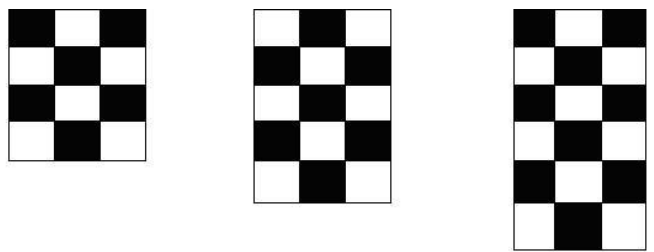

Fig. 8. Chessboard as specific point

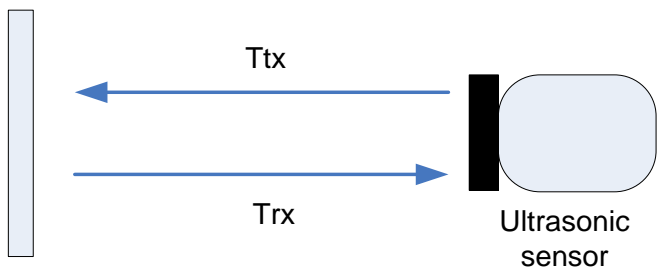

Fig. 9. Ultrasonic sensor, distance is measured by converting half of Ttx transmitting time and Trx receiving time

\section{Best path finding}

When EWC detect the obstacle, it must understand where the best path should be chosen if EWC want to go into specific place. Best path will be chosen based on floor layout map and image map. Image map is created by acquiring background images in every $1 \mathrm{~m}$ as is shown in Figure 10. In every location ( $\mathrm{x}, \mathrm{y})$ have one image. Instance, if the location has size $10 \times 10 \mathrm{~m}$, it will have 100 images. After acquire background images, floor layout map will be added manually as initial condition. This map is setup manually based on room layout. Example is shown in Figure 11.

Combination between background images and layout path will obtain main map. EWC will move consider the main map. After obstacle is detected in current path, EWC will switch to another path which have same destination. Obstacle avoidance methods are also useful when user is not confident to pass the obstacle. EWC will take over and pass the obstacle. Best path is chosen based on Dijkstra algorithm. Furthermore, System also able to renew existing map.
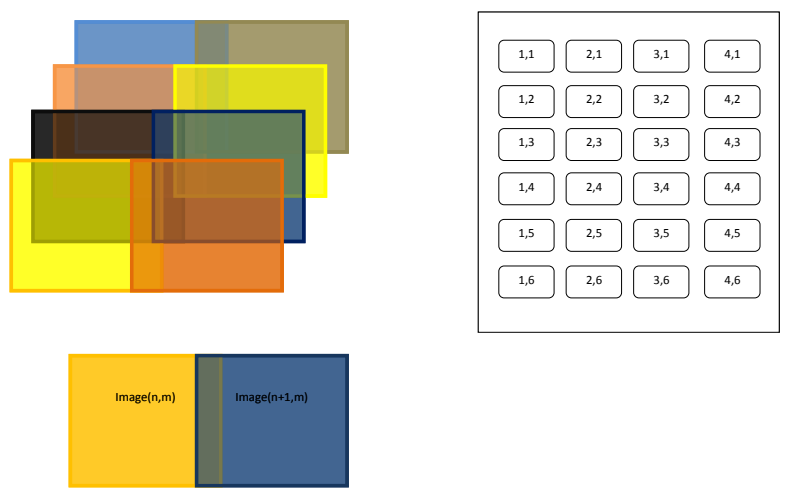

Fig. 10. Image map, created by acquire image in every $1 \mathrm{~m}$. 


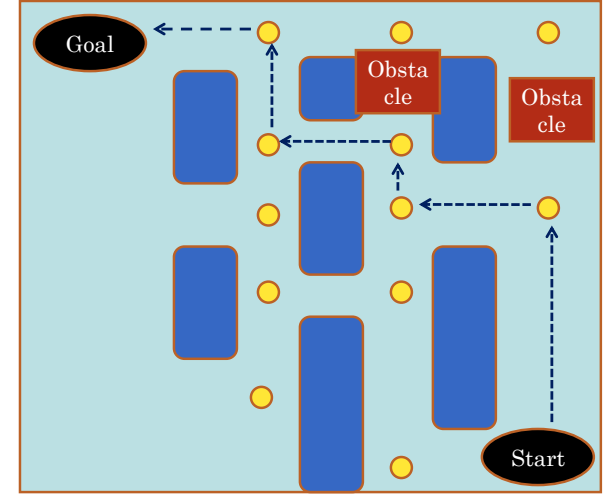

Fig. 11. Best path finding using Dijkstra, best path is chosen based on shortest distance

\section{EXPERIMENTS}

\section{A. Key selection}

The proposed method experiments by measuring success rate eye gaze detection as hit keys selection. Success rate of eye gaze detection is measured by change user distance to camera. Experiments say that minimum distance of success rate is $13 \mathrm{~cm}$ and maximum distance is $38 \mathrm{~cm}$. The distance beyond these, OpenCV will not detect the eye and caused the system fail to measure eye gaze. Hit keys selection experiment was done by using real time video 640 by 480 pixels. Data experiments when measure hit keys selection on range distance $24-30 \mathrm{~cm}$ is shown in Table 1 .

TABLE I. HIT KEYS MEASUREMENT

\begin{tabular}{|c|c|c|c|c|}
\hline & \multicolumn{4}{|c|}{ Success Rate } \\
\cline { 2 - 5 } $\begin{array}{c}\text { Distance } \\
(\mathbf{c m})\end{array}$ & $\begin{array}{c}\text { Stop } \\
(\boldsymbol{\%})\end{array}$ & $\begin{array}{c}\text { Move Forward } \\
(\boldsymbol{\%})\end{array}$ & $\begin{array}{c}\text { Turn } \\
\text { Left } \\
(\boldsymbol{\%})\end{array}$ & $\begin{array}{c}\text { Turn } \\
\text { Right } \\
(\boldsymbol{\%})\end{array}$ \\
\hline $\mathbf{2 4}$ & 100 & 100 & 100 & 100 \\
$\mathbf{2 5}$ & 100 & 100 & 100 & 100 \\
$\mathbf{2 6}$ & 100 & 100 & 100 & 100 \\
$\mathbf{2 7}$ & 100 & 100 & 100 & 100 \\
$\mathbf{2 8}$ & 100 & 100 & 100 & 100 \\
$\mathbf{2 9}$ & 100 & 100 & 100 & 100 \\
$\mathbf{3 0}$ & 94.12 & 100 & 100 & 100 \\
\hline
\end{tabular}

The result of hit key measurement is shown almost perfectly, Error which cannot understand the eye input is caused by the system fail to verify the template image. Error will zero if good quality template is used. To make sure that good quality template is used; System will always verify every template and analyzed it. If templates have poor quality, system will conduct calibration step again. This step will repeat until good quality template is obtained.

\section{B. Obstacle avoidance performance.}

Experiment of obstacle avoidance is conducted by acquiring image in corridor with distance $1 \mathrm{~m}$ per images. Location which image was acquired, is set with $(\mathrm{x}, \mathrm{y})$ coordinate. So, in every $(\mathrm{x}, \mathrm{y})$ location will have 1 background image. This image will be used as background reference. Obstacle is detected by subtract background image with current image. To eliminate problem which caused by different position between these images, affine transformation is used to transform background image to affine image which have same position with current image. Applying affine transformation will does work if only if three noticeable important points are appears on both images. These important points are represented by three types of chessboard. If the chessboard is successful detected, then by using affine transformation and subtraction between background image and current image, obstacle is founded. Experiments of chessboard detection is conducted by measuring maximum location which still detected by system. Chessboard is put in fix location. After that, EWC is move around the chessboard. Maximum location which detected by system is recorded. Data experiments are shown in Figure 12.
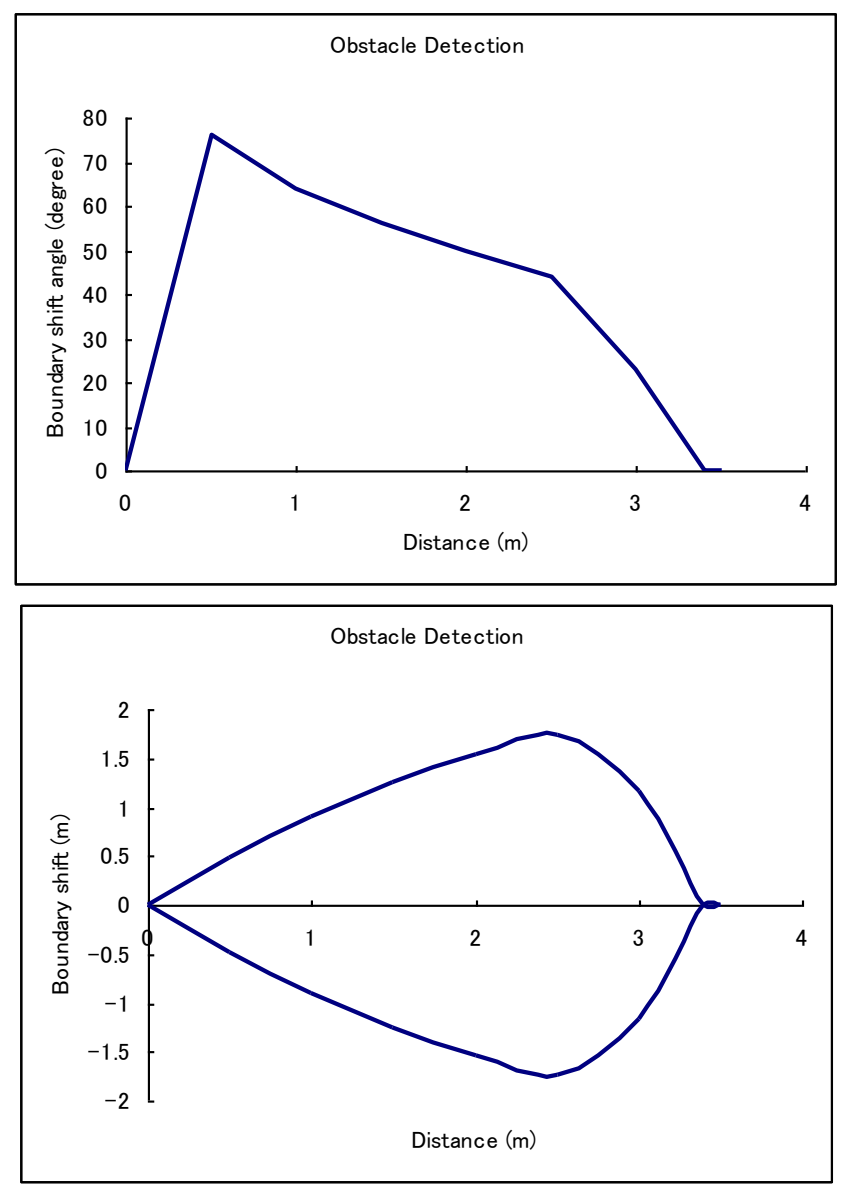

Fig. 12. measuring obstacle avoidance performances

Data show that boundary shift will decrease when distance between camera and chessboard is increase. This experiment is equal to obstacle detection. After three types of chessboard are detected, affine transformation will use these points (three chessboard center of areas) to subtract with current image and obstacle position will be found.

\section{Ultrasonic Sensor}

Objective of this experiment is measure accuracy of ultrasonic sensor before used in the system. This experiment is conducted by measure sensor output for measuring distance and comparing with real data. Some object is put on front of sensor with varies distances and measure it. The experiment is conducted on distance $0 \mathrm{~cm}$ until $3 \mathrm{~m}$. 
Ultrasonic sensor use PING type parallax product and microcontroller AT89S51 as processing data (convert from time value to distance output). Graph of accuracy sensor is shown in Figure 13.

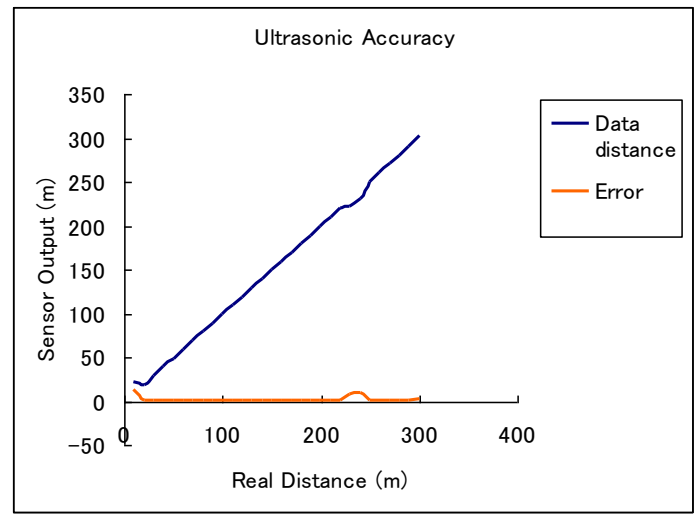

Fig. 13. Experiment of ultrasonic accuracy. This shown that minimum distance is around $3 \mathrm{~cm}$ and maximum distance is $3 \mathrm{~m}$. This range is appropriate for detect the object.

Elevation angle is require to know how width of the beam sensor. Ultrasonic sensor with width beam is not benefit to our system. Narrow beam will obtain good result because it will not influence with any disturbance. This experiment is conducted by measure elevation angle from $0 \mathrm{~cm}$ until $3 \mathrm{~m}$. Graph of elevation angle is shown in Figure 14.
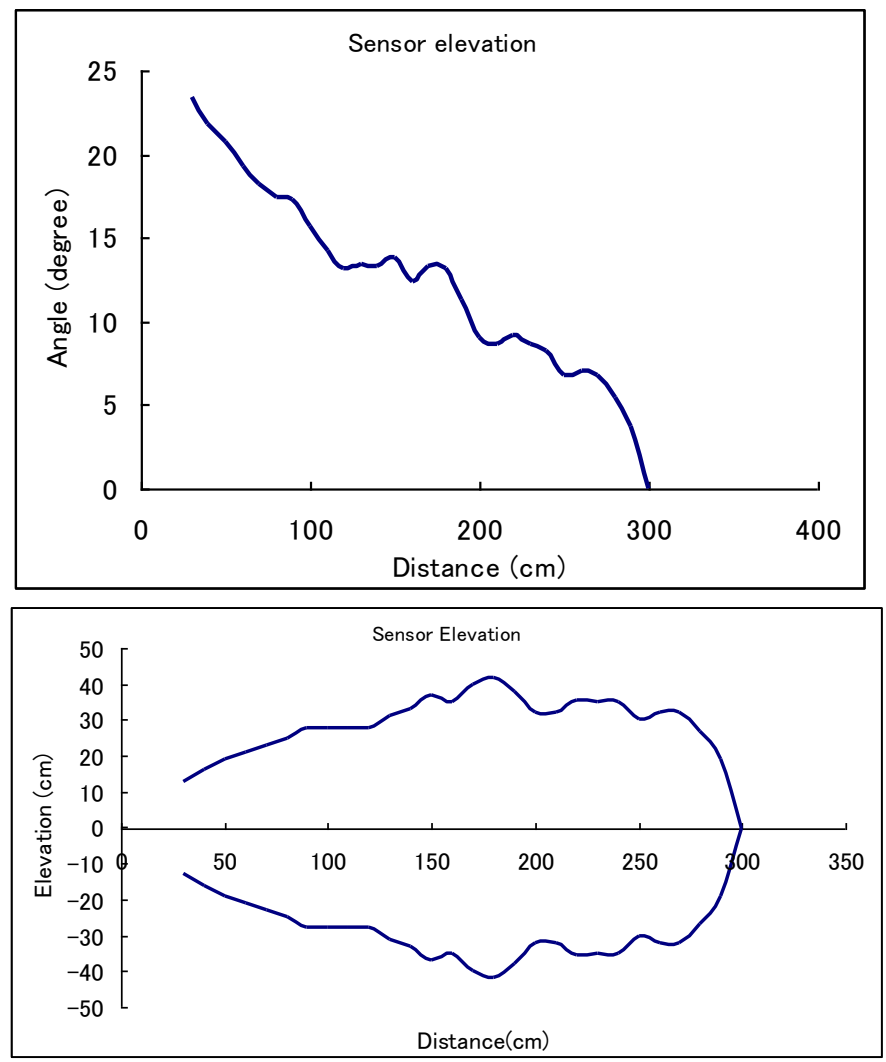

Fig. 14. Experiment of Elevation angle. Top side is distance versus angle, and bottom side is distance versus distance elevation.

\section{Performance of Electric Wheel Chair}

This experiment is conducted for measure EWC performance on starting up acceleration, forward and backward breaking deceleration. Also conducted speed measurement when EWC move forward, backward, turn left, and turn right. EWC is drive by user who has weight is $73 \mathrm{~kg}$. We record the duration time and convert it into speed value. Experiment data of speed measurement is shown in Table 2. Graph of EWC acceleration and deceleration when start and stop duration is shown in Figure 15.

TABLE II. SPEED MEASUREMENT

\begin{tabular}{|c|c|}
\hline Moving & Speed $(\mathbf{m} / \mathbf{s})$ \\
\hline Forward & 0.29 \\
Backward & 0.26 \\
Turn Left & 0.11 \\
Turn Right & 0.11 \\
\hline
\end{tabular}
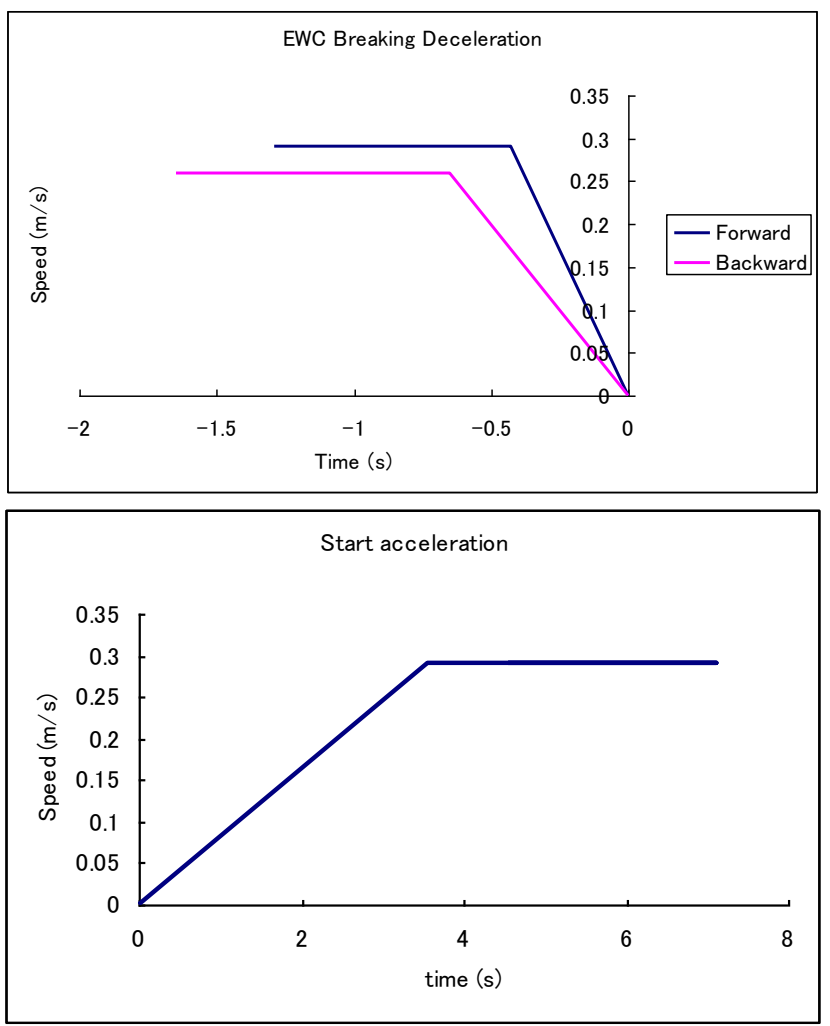

Fig. 15. Experiment of EWC acceleration and deceleration

\section{E. Processing time each process}

In order to apply whole method into EWC application, processing time should be measured to identify performance of our real time system. Figure 16 shows transient time of eye detection and tracking, $\mathrm{n}$ the beginning of chart, it seem this method take long time around $300 \mathrm{~ms}$. In this time, system still process face detection, eye detection and others process before running template matching method. After eye location is founded, then system bypass previous step and cause process working fast. 
Meanwhile, Figure 17 shows processing time of eye detection and tracking on steady state condition, it looks faster than transient condition.

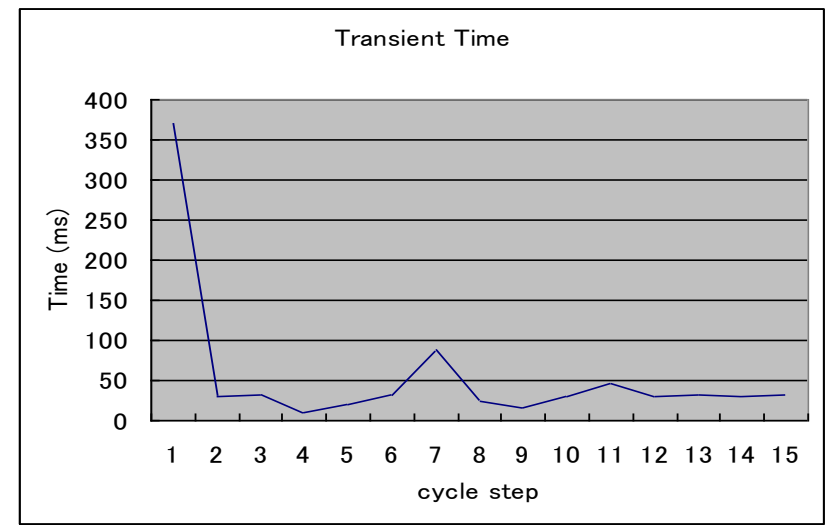

Fig. 16. Transient time of eye detection and tracking, $n$ the beginning of chart, it seem this method take long time around $300 \mathrm{~ms}$. In this time, system still process face detection, eye detection and others process before running template matching method. After eye location is founded, then system bypass previous step and cause process working fast.

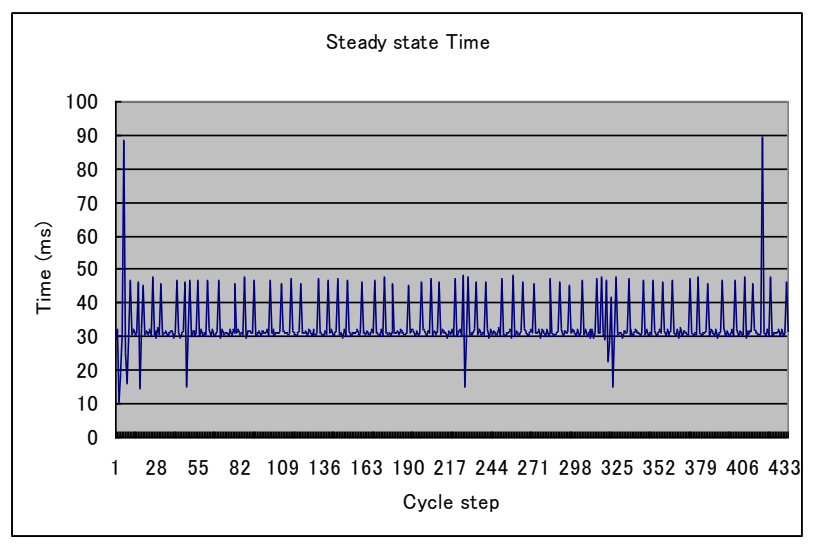

Fig. 17. Processing time of eye detection and tracking on steady state condition, it looks faster than transient condition.

\section{F. Eye detection and tracking}

This experiment is conducted using Optiplex 755 Dell computer with Intel Core 2 Quad CPU $2.66 \mathrm{GHz}$ and $2 \mathrm{G}$ of RAM. We use NET COWBOY DC-NCR131 camera as visual input. Experimental result show average steady state processing time is $32.625 \mathrm{~ms}$. it also shows difference processing time between transient and steady state condition. Transient time require more time than steady state time.

\section{G. Eye gaze measurement}

Objective of this experiment is measure processing time on Eye gaze identification. It is conducted by using ACER computer ASPIRE 5572 Series Core Duo T2050 1.6 GHz CPU and $1 \mathrm{G}$ of RAM. Result data show average processing time of this method is $342.379 \mathrm{~ms}$. Figure 18 shows processing time of Eye gaze method.

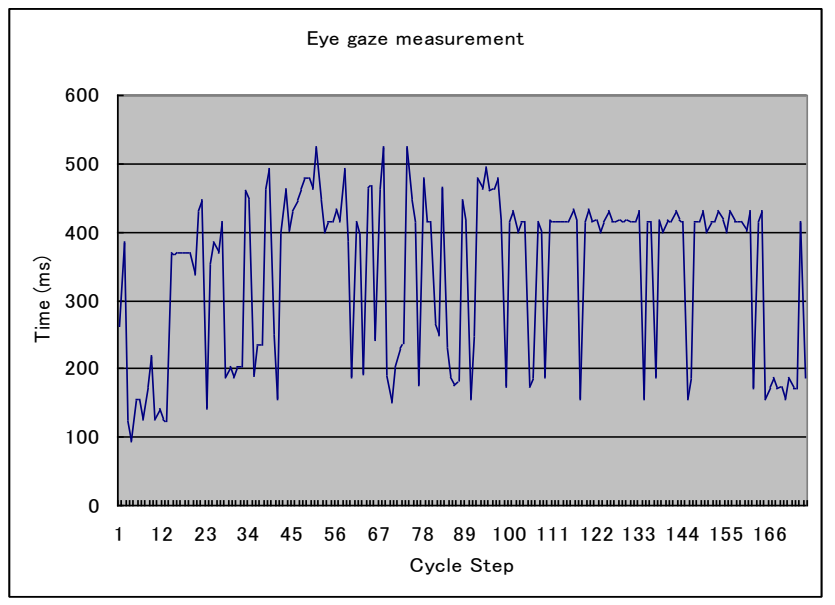

Fig. 18. processing time of Eye gaze method

\section{H. Obstacle detection}

This experiment also was conducted using Optiplex 755 Dell computer with Intel Core 2 Quad CPU $2.66 \mathrm{GHz}$ and 2G of RAM. NET COWBOY DC-NCR131 camera as visual input is also used. Experimental result show average processing time is $32.625 \mathrm{~ms}$. Figure 19 shows processing time of obstacle detection.

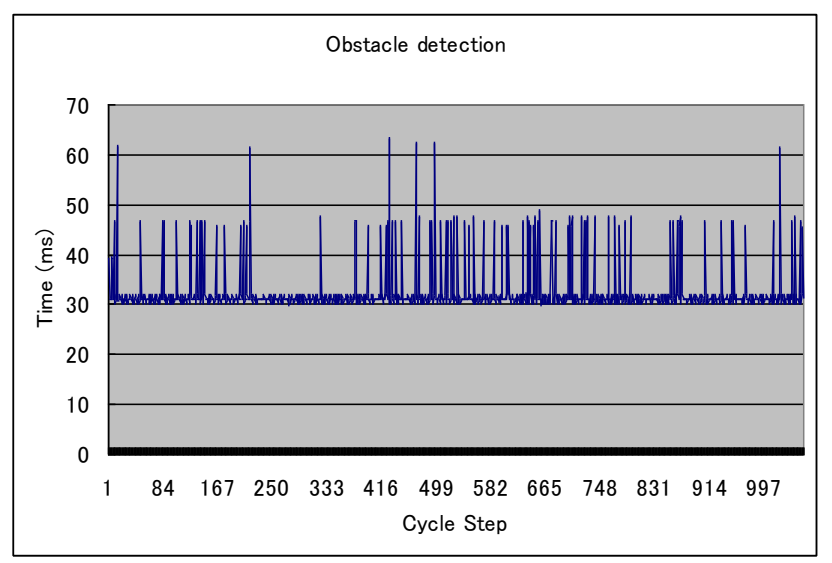

Fig. 19. processing time of obstacle detection

\section{Ultrasonic sensor}

We implemented ultrasonic sensor parallax PING type. This sensor is controlled by using custom microcontroller AT89S51. Data was stored into computer by using USB communication. Result data show average processing time is $568.658 \mathrm{~ms}$. Figure 20 also shows processing time of ultrasonic sensor, it look take longer time than others. 


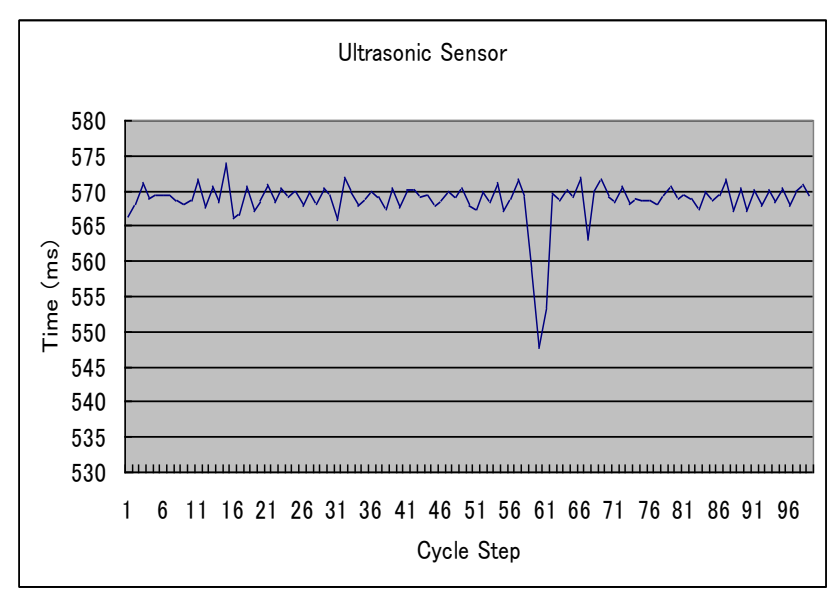

Fig. 20. processing time of ultrasonic sensor, it look take longer time than others.

\section{CONCLUSION}

It is concluded that the proposed key-in system with human eyes only works $100 \%$ perfectly for the number of keys is four, start, stop, turn right and left. Also it is concluded that the proposed EWC control system does work in a real basis avoiding obstacles on range bellow $3.4 \mathrm{~m}$ using image processing method and bellow $3 \mathrm{~m}$ using ultrasonic sensor. By the proposed system, EWC is able to identify obstacle and avoid them. Obstacle avoidance can assist user into interest place although undesired condition such as dark areas, glass wall or door, smoke area, and etc. By implemented this system, EWC will move more safely and comfortable.

\section{ACKNOWLEDGMENT}

The author would like to thank Dr.Djoko of Institute of Technology Surabaya, Indonesia for his efforts through experiments and simulations.

\section{REFERENCES}

[1] Park, K.S., and Lee K.T., Eye controlled human/computer interface using the line of sight and intentional blink, Computer Ind. Eng., 30, 3, 463-473, 1996 .

[2] Ito, Sudo, the Ifuku part: "The look input type communication equipment for serious physically handicapped persons", the Institute of Electronics, Information and Communication Engineers paper magazine, J83D1， 5, 495.503, 2000.

[3] Yamada, Fukuda: "The text creation and the peripheral equipment control device" by eye movement, the Institute of Electronics, Information and Communication Engineers paper magazine , J69D, 7, 1103-1107, 1986.
[4] Mitsunori Yamada, the research trend of the latest eye movement, an electric information-and-telecommunications academic journal, MBE95132, NC95-90, 145-152, 1995.

[5] K.Abe, S.Ohiamd M.Ohyama: "An Eye-gaze Input System based on the Limbus Tracking Method by Image Analysis for Seriously Physically Handicapped People", $7^{\text {th }}$ ERCIM Workshop "User Interface for All" Adjunct Proc., 185-186, 2002.

[6] http://www.creact.co.jp/jpn/por.pdf

[7] Kuno, Yagi, Fujii, Koga, Uchikawa: "Development of the look input interface using EOG", the Information Processing Society of Japan paper magazine, 39, 5, 1455-1462, 1998.

[8] D.A.Robinson: A method of measuring eye movement using a sclera search coil in a magnetic field, IEEE Trans. on Biomedical Electronics, 10, 137-145, 1963.

[9] http://webvision.med.utah.edu/

[10] Ito, Nara: "Eye movement measurement by picture taking in and processing via a video capture card", an Institute of Electronics, Information and Communication Engineers technical report, 102, 128 , 31-36, 2002.

[11] Kishimoto, Yonemura, Hirose, Changchiang: "Development of the look input system by a cursor move system", and the Institute of Image Information and Television Engineers, 55， 6， 917-919, 2001.

[12] Corno, L.Farinetti, I. Signorile: "A Cost-Effective Solution for EyeGaze Assistive Technology", Proc. IEEE International Conf. on Multimedia and Expo, 2, 433-436, 2002.

[13] Abe, Ochi, Oi, Daisen: "The look input system using the sclera reflection method by image analysis", and the Institute of Image Information and Television Engineers, 57, 10, 1354-1360, 2003.

[14] Abe, Daisen, Oi: "The multi-index look input system which used the image analysis under available light", and the Institute of Image Information and Television Engineers, 58, 11, 1656-1664, 2004.

[15] Abe, Daisen, Oi: "The look input platform for serious physically handicapped persons", Human Interface Society Human interface symposium 2004 collected papers, 1145-1148, 2004.

[16] http://homepages.inf.ed.ac.uk/rbf/CVpnline/LOCAL_COPIES/GONG1/ cvOnline-skinColourAnalysis.html.

[17] Gary Bradski, Andrian Kaebler, "Learning Computer Vision with the OpenCV Library", O'REILLY, 214-219, 2008.

\section{AUTHORS PROFILE}

Kohei Arai, He received BS, MS and PhD degrees in 1972, 1974 and 1982, respectively. He was with The Institute for Industrial Science and Technology of the University of Tokyo from April 1974 to December 1978 also was with National Space Development Agency of Japan from January, 1979 to March, 1990. During from 1985 to 1987, he was with Canada Centre for Remote Sensing as a Post Doctoral Fellow of National Science and Engineering Research Council of Canada. He moved to Saga University as a Professor in Department of Information Science on April 1990. He was a councilor for the Aeronautics and Space related to the Technology Committee of the Ministry of Science and Technology during from 1998 to 2000. He was a councilor of Saga University for 2002 and 2003. He also was an executive councilor for the Remote Sensing Society of Japan for 2003 to 2005. He is an Adjunct Professor of University of Arizona, USA since 1998. He also is Vice Chairman of the Commission "A" of ICSU/COSPAR since 2008. He wrote 30 books and published 478 journal papers 